\title{
Übergangsmetall-katalysierte Additionsreaktionen von 3-Phenyl-2H-azirinen und Acetylencarbonsäureestern
}

\author{
Inada, Akira ; Heimgartner, Heinz
}

\begin{abstract}
Transition Metal Catalyzed Addition Reactions of 3-Phenyl-2H-azirines and Alkyl Acetylene Carboxylates. In the presence of molybdenum hexacarbonyl, the 3-phenyl-2H-azirines 1 and 7 react with alkyl acetylene carboxylates 2 via the cleavage of the $\mathrm{C}, \mathrm{N}$-double bond to give $2 \mathrm{H}$-pyrroles 5 or pyrrole 9 (Table), whose structures were deduced from the spectra data, in particular 13C-NMR data. The $2 \mathrm{H}$-pyrrole $5 \mathrm{a}$ was also obtained by treatment of a mixture of 1 and $2 \mathrm{a}$ with tungsten hexachloride. A tentative mechanism for the formation of the $2 \mathrm{H}$-pyrroles is formulated.
\end{abstract}

DOI: https://doi.org/10.1002/hlca.19820650523

Posted at the Zurich Open Repository and Archive, University of Zurich ZORA URL: https://doi.org/10.5167/uzh-97546

Journal Article

Originally published at:

Inada, Akira; Heimgartner, Heinz (1982). Übergangsmetall-katalysierte Additionsreaktionen von 3Phenyl-2H-azirinen und Acetylencarbonsäureestern. Helvetica Chimica Acta, 65(5):1489-1498.

DOI: https://doi.org/10.1002/hlca.19820650523 


\title{
147. Übergangsmetall-katalysierte Additionsreaktionen von 3-Phenyl-2 $\boldsymbol{H}$-azirinen und Acetylencarbonsäureestern
}

\author{
von Akira Inada ${ }^{1}$ ) und Heinz Heimgartner ${ }^{2}$ ) \\ Organisch-chemisches Institut der Universität Zürich, Winterthurerstrasse 190, CH-8057 Zürich
}

(18.1II. 82)

Transition Metal Catalyzed Addition Reactions of 3-Phenyl-2H-azirines and Alkyl Acetylene Carboxylates

\section{Summary}

In the presence of molybdenum hexacarbonyl, the 3-phenyl-2 $\mathrm{H}$-azirines 1 and 7 react with alkyl acetylene carboxylates 2 via the cleavage of the $\mathrm{C}, \mathrm{N}$-double bond to give $2 \mathrm{H}$-pyrroles 5 or pyrrole 9 (Table), whose structures were deduced from the spectra data, in particular ${ }^{13} \mathrm{C}-\mathrm{NMR}$. data. The $2 \mathrm{H}$-pyrrole $5 \mathbf{a}$ was also obtained by treatment of a mixture of $\mathbf{1}$ and $\mathbf{2 a}$ with tungsten hexachloride. A tentative mechanism for the formation of the $2 H$-pyrroles is formulated.

Im Zusammenhang mit Untersuchungen zur Reaktivität von $2 \mathrm{H}$-Azirinen haben wir vor einiger Zeit auch metallcarbonyl-katalysierte Additionsreaktionen mit Acetylenen studiert. Bei der Umsetzung von 2,2-Dimethyl-3-phenyl-2 $\mathrm{H}$-azirin (1) mit Dieisenenneacarbonyl in Gegenwart von überschüssigem Acetylendicarbonsäure-dimethylester (2a) bildeten sich u. a. zwei neue Dieisen-hexacarbonylkomplexe, denen aufgrund von Röntgenstrukturanalysen die Strukturen 3 [1] und 4 [2] zukommen (Schema 1). Als Primärschritt dieser Reaktion ist eine Komplexierung des Azirin-N-Atoms, gefolgt von Spaltung der C,N-Einfachbindung unter Ausbildung eines Vinylnitren-eisentetracarbonyl-Komplexes formuliert worden [1] (vgl. dazu auch [3] und dort zit. Lit.).

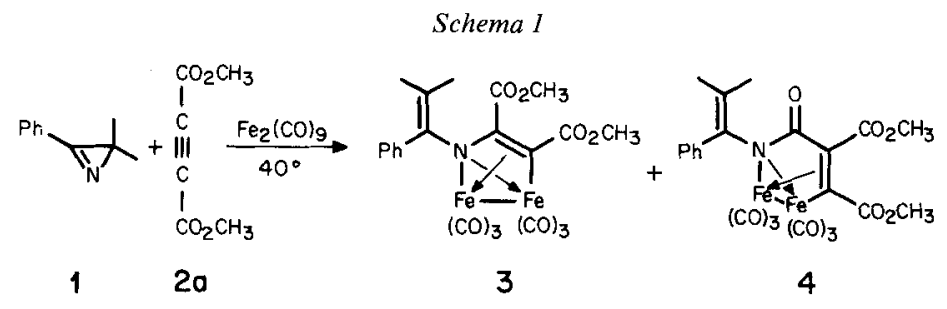

\footnotetext{
1) Neue Adresse: Pharmaceutical Sciences of Josai University, 1-1 Keyakidai, Sakado, Saitama 350-02, Japan.

2) Korrespondenzautor.
} 
Einen ganz anderen Verlauf nimmt die Umsetzung des Azirins 1 und Acetylendicarbonsäure-dimethylester in Gegenwart von Molybdänhexacarbonyl. Über diese Reaktionen ist schon in einer vorläufigen Mitteilung berichtet worden [4]; die vorliegende Arbeit enthält ergänzende Versuche und den vollständigen experimentellen Teil.

Molybdänhexacarbonyl-induzierte Additionen von 3-Phenyl-2 $\mathrm{H}$-azirinen und Acetylencarbonsäureestern. - Eine Lösung von 2,2-Dimethyl-3-phenyl-2 $\mathrm{H}$-azirin (1) und Acetylendicarbonsäure-dimethylester (2a) sowie $\mathrm{Mo}(\mathrm{CO})_{6}$ (Molverhältnis ca. $1: 2: 1)$ in getrocknetem Benzol wurde unter Ar-Atmosphäre drei Tage bei $35-45^{\circ}$ gerührt. Nach Filtration und wiederholter Chromatographie an Kieselgel wurde 2,2-Dimethyl-3-phenyl-2 $\mathrm{H}$-pyrrol-4,5-dicarbonsäure-dimethylester (5a) in $28 \%$ Ausbeute erhalten (Schema 2, Tab.). Bei dieser Ringerweiterung vom 3- zum 5-gliedrigen Heterocyclus wird offensichtlich die C, N-Doppelbindung des Azirins 1 gespalten, im Gegensatz zu der in Schema 1 skizzierten $\mathrm{Fe}_{2}(\mathrm{CO})_{9}$-katalysierten Additionsreaktion, die unter Spaltung der $\mathrm{C}, \mathrm{N}$-Einfachbindung verläuft.

In Schema 2 ist der $\mathrm{Mo}(\mathrm{CO})_{6}$-katalysierten Additionsreaktion von 1 und 2 die photochemisch induzierte gegenübergestellt, die im Falle von 2a unter C,CBindungsbruch zum $2 \mathrm{H}$-Pyrrol $\mathbf{6 a}$, einem Isomeren von 5a, führte [5]. Die zu 5a analogen $2 \mathrm{H}$-Pyrrole $\mathbf{5 b}$ und $\mathbf{5 c}$ wurden aus dem Azirin 1 und Acetylendicarbonsäure-diäthylester (2b) bzw. Propiolsäure-methylester (2c) in Gegenwart von $\mathrm{Mo}(\mathrm{CO})_{6}$ gebildet $(\mathrm{Tab}$.). Unter gleichen Reaktionsbedingungen entstand hingegen aus 1 und Phenylpropiolsäure-äthylester (2d) kein $2 \mathrm{H}$-Pyrrol; als einziges Produkt wurde das Dimerisierungsprodukt des Azirins isoliert, nämlich 2,2,5,5-Tetramethyl3,6-diphenyl-2, 5-dihydropyrazin (8) [6].

Bei der $\mathrm{Mo}(\mathrm{CO})_{6}$-katalysierten Reaktion von 1 und Propiolsäure-methylester (2c) bildete sich - allerdings in geringer Ausbeute - regioselektiv das $2 \mathrm{H}$-Pyrrol 5c (Tab.); isomere Produkte konnten nicht nachgewiesen werden ${ }^{3}$ ).

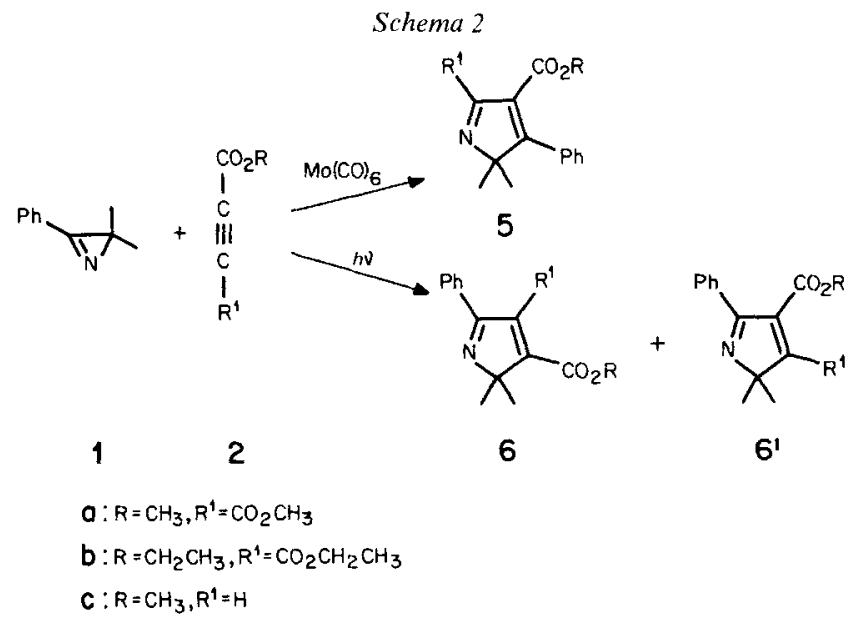

$\left.{ }^{3}\right)$ Aus dem Reaktionsgemisch wurden neben $5 \mathbf{c}$ geringe Mengen (E,E)-2,2'-Oxydiacrylsäuredimethylester (11) [7] isoliert (s. Exper. Teil). 
Tabelle. Molybdänhexacarbonyl-induzierte Reaktionen von 3-Phenyl-2H-azirinen mit Acetylencarbonsäureestern

2H-Azirin

Die Umsetzung von 2,3-Diphenyl-2 $H$-azirin (7) mit dem Acetylen 2a in Gegenwart von $\mathrm{Mo}(\mathrm{CO})_{6}$ lieferte in $20 \%$ Ausbeute 4,5-Diphenylpyrrol-2,3-dicarbonsäuredimethylester (9 [8]), ein Isomeres des photochemisch erzeugten Pyrrols 12 [9] (Schema 3). Die Bildung von 9 kann in Analogie zur vorangehend beschriebenen Reaktion mit dem Azirin 1 über die primäre Erzeugung des 2,3-Diphenyl-2 $\mathrm{H}$ pyrrol-4,5-dicarbonsäure-dimethylesters (b) und anschliessende Tautomerie formuliert werden (Schema 3).

In Abwesenheit der Acetylencarbonsäureester wurden aus den Azirinen 1 und 7 in Gegenwart von $\mathrm{Mo}(\mathrm{CO})_{6}$ das schon früher beschriebene Dimere 8 bzw. das «Dehydro-Dimere», nämlich 2,3,5,6-Tetraphenylpyrazin (10 [10], Tab.) erhalten. 
Schema 3

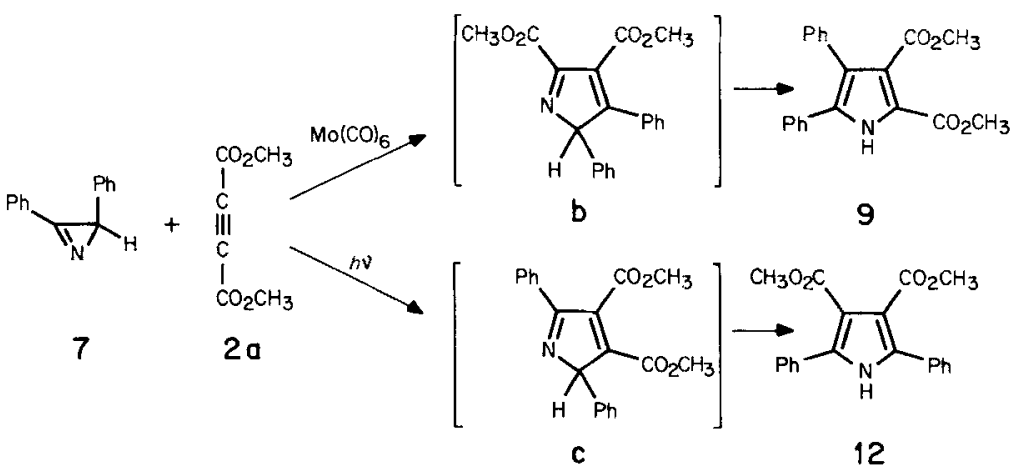

Infolge der symmetrischen Strukturen dieser Produkte kann nicht entschieden werden, welche der ursprünglichen Azirinbindungen bei dieser Dimerisierung gebrochen wird ${ }^{4}$ ).

Die Strukturen der gebildeten (1:1)-Addukte (MS., Elementaranalysen; s. Exper. Teil) wurden mit Hilfe der spektroskopischen Daten bestimmt:

Im Falle des (1:1)-Adduktes aus 1 und 2a liess sich die Struktur des $2 H$-Pyrrols 6a durch direkten Vergleich mit dem auf photochemischem Wege hergestellten Material [5] ausschliessen. Zwischen den beiden verbleibenden, alternativen Strukturen 5a und d (Schema 4) liess sich mit Hilfe der ${ }^{13} \mathrm{C}$-NMR.-Spektroskopie entscheiden. Während die gemessene chemische Verschiebung von $82,3 \mathrm{ppm}$ für das $\mathrm{sp}^{3}$-hybridisierte, quartäre $\mathrm{C}$-Atom gut mit den für das $\mathrm{C}(2)$-Atom der $2 H$-Pyrrole 13 [12] sowie 6a, 14 und 15 (s. Exper. Teil) ermittelten Werten übereinstimmt (Schema 4), müsste für C(3) im isomeren $3 H$-Pyrrol d ein Signal bei deutlich höherem Feld erwartet werden. So absorbiert C(3) im 2,3,3-Trimethyl-3H-indol (16) [13] bei $53,5 \mathrm{ppm}$ und im $3 H$-Pyrrol 17 [14] (Schema 4) bei

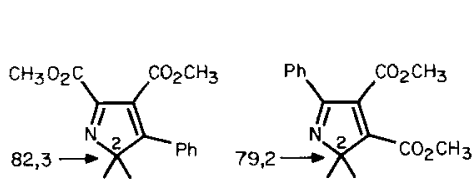

50

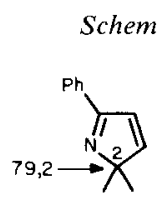

$13[12]$

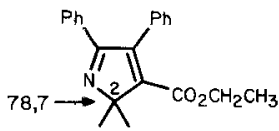

14

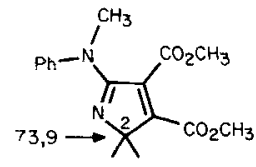

15

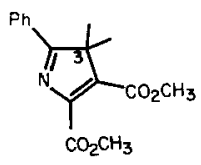

$d$

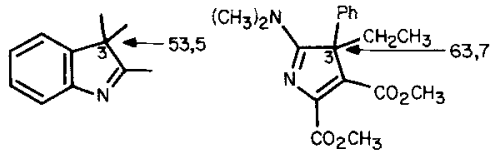

16 [13] 17 [14]

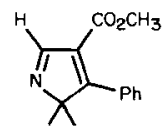

$5 c$

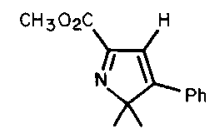

e

${ }^{4}$ ) Ein Bildungsmechanismus unter Spaltung der Azirin-C,C-Bindung ist in [11] postuliert worden. 
63,7 ppm. Somit muss dem (1:1)-Addukt aus der $\operatorname{Mo}(\mathrm{CO})_{6}$-katalysierten Reaktion von 1 und 2a die Struktur 5a zugeschrieben werden. In Analogie zu den Strukturen 5a und $5 \mathbf{b}$ kommt dem durch die $\mathrm{Mo}(\mathrm{CO})_{6}$-katalysierte Reaktion aus 1 und $2 \mathrm{c}$ in nur geringer Ausbeute gebildeten (1:1)-Addukt entweder die Struktur 5c oder e zu (Schema 4). Eine Entscheidung zwischen diesen beiden Formeln ermöglicht das ${ }^{1} \mathrm{H}-\mathrm{NMR}$.-Spektrum: Das «Azomethin-H» $(\mathrm{H}-\mathrm{C}(5))$ erscheint bei 8,15 ppm, was für die Struktur des 2,2-Dimethyl-3-phenyl-2 $H$-pyrrol-4-carbonsäuremethylester (5c) spricht. Im isomeren $2 \mathrm{H}$-Pyrrol e müsste $\mathrm{H}-\mathrm{C}$ (4) dagegen bei $\mathrm{ca}$. 7,3 ppm absorbieren (vgl. dazu $\mathrm{H}-\mathrm{C}$ (4) in 6c (Schema 2): 7,29 ppm [15]).

Die Struktur des aus 2,3-Diphenyl-2 $\mathrm{H}$-azirin (7) und 2a gebildeten Pyrrols 9 (Schema 3) folgt aus den spektralen Daten und dem Smp.-Vergleich mit dem Literaturwert [8]. Dass es sich nicht um die symmetrische Struktur des 2,5-Diphenylpyrrol-3,4-dicarbonsäure-dimethylesters (12) [9] handeln kann, ist aus dem IR.-Spektrum (zwei CO-Banden bei 1735 und $1685 \mathrm{~cm}^{-1}$ ) und dem ${ }^{1}$ H-NMR.-Spektrum (zwei $\mathrm{CH}_{3} \mathrm{O}$-Signale bei 3,85 und 3,75 ppm) ersichtlich.

Umsetzung von 2,2-Dimethyl-3-phenyl-2 $\mathrm{H}$-azirin mit Acetylendicarbonsäuredimethylester in Gegenwart von Wolframhexachlorid und Tetrabutylzinn. - Im Laufe unserer Untersuchungen über metall-katalysierte Reaktionen von $2 \mathrm{H}$-Azirinen interessierte uns auch der Vergleich der katalytischen Wirkung von $\mathrm{Mo}(\mathrm{CO})_{6}$ und anderen Übergangsmetallen. Aufgrund mechanistischer Überlegungen wurde unter anderem auch Wolframhexachlorid eingesetzt, welches als effizienter Katalysator für Metathesisreaktionen verwendet worden ist $[16]^{5}$ ). Die Umsetzung des $2 \mathrm{H}$ Azirins 1 mit Acetylendicarbonsäure-dimethylester (2a) in Trichloräthylen bei $45-50^{\circ}$ lieferte in Gegenwart von $\mathrm{WCl}_{6} /\left(\mathrm{C}_{4} \mathrm{H}_{9}\right)_{4} \mathrm{Sn}[18]$ das $2 \mathrm{H}$-Pyrrol 5a in $12 \%$ Ausbeute.

Diskussion. - Ein Reaktionsmechanismus für die $\mathrm{Mo}(\mathrm{CO})_{6}$-katalysierte Bildung von $2 H$-Pyrrolen bzw. Pyrrolen via Spaltung der Azirin-C, N-Doppelbindung ist in Schema 5 vorgeschlagen. Danach bildet das Azirin primär einen Molybdänpentacarbonyl-Komplex (vgl. z. B. [11] [19]) und anschliessend unter erneutem CO-Verlust zusammen mit dem Acetylen den $\pi$-Komplex f. Kupplungsreaktion zwischen dem Acetylen und dem Azirin unter Bildung einer C,C-Bindung liefert das Molybdapyrrolin g (vgl. z. B. [20]). Aus diesem entsteht dann unter Dekom-

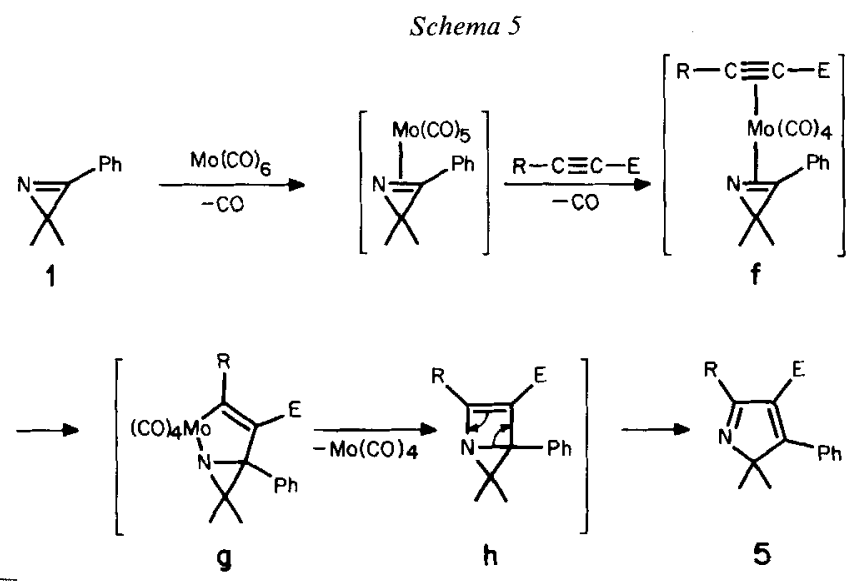

5) In einigen Fällen wurde auch $\mathrm{Mo}(\mathrm{CO})_{6}$ als Katalysator für Metathesisreaktionen eingesetzt [17]. 
plexierung das 1-Azabicyclopenten h bzw. das $2 \mathrm{H}$-Pyrrol 5. Die Bildung des $2 \mathrm{H}$ Pyrrols 5a in Gegenwart von $\mathrm{WCl}_{6} /\left(\mathrm{C}_{4} \mathrm{H}_{9}\right)_{4} \mathrm{Sn}$ verläuft wahrscheinlich nach einem ähnlichen Mechanismus. Ebenfalls mit einem analogen Reaktionsverlauf kann die leichte, $\mathrm{Ni}$ (II)-katalysierte Bildung von Pyrrolen aus $2 \mathrm{H}$-Azirinen und enolisierbaren Ketonen beschrieben werden [21] (Schema 6).

Die voranstehend diskutierten Reaktionen zu $2 \mathrm{H}$-Pyrrolen und Pyrrolen verlaufen offensichtlich unter Spaltung der ursprünglichen Azirin-C,N-Doppelbindung $^{6}$ ). Es ist möglich, dass auch die Bildung des Dihydropyrazins 8 und des Pyrazins 10 via die gleiche Azirinringöffnung verläuft; allerdings kann mit den bis jetzt vorliegenden Daten die früher postulierte, alternative Bildung der Dimeren unter Spaltung der Azirin-C,C- oder Azirin-C, N-Einfachbindung [11] [19] nicht ausgeschlossen werden.

Die Übergangsmetall-katalysierte Umsetzung von $2 H$-Azirinen mit Acetylenen stellt einen neuen Zugang zu $2 H$-Pyrrolen dar. Offenbar handelt es sich bei 2,2disubstituierten $2 \mathrm{H}$-Azirinen ganz allgemein um geeignete Vorläufer für $2 \mathrm{H}$-Pyrrole, einen relativ schlecht zugänglichen Heterocyclus-Typ [27]: So führt z.B. die 1,3-dipolare Cycloaddition der aus $2 \mathrm{H}$-Azirinen photochemisch erzeugten Nitrilylide mit Acetylenen zu $2 \mathrm{H}$-Pyrrolen [5] [15] [28]. Auch die entsprechenden photochemischen Umsetzungen in Gegenwart von Vinylphosphoniumsalzen [12] [29], Vinylsulfonen [12] und $a$-Äthoxyacrylonitril [12] liefern über einen Additions/ Eliminierungsmechanismus $2 \mathrm{H}$-Pyrrole. Eine weitere ergiebige Synthese der Heterocyclen vom Typ 19 wurde in der Reaktion von 2,2-disubstituierten $2 \mathrm{H}$ Azirinen 18 mit Enolat-Ionen beschrieben [30] (Schema 6). Es ist bemerkenswert, dass diese unter Spaltung der Azirin-C,N-Doppelbindung verlaufende, basenkatalysierte Reaktion eine Parallele zur Ni(II)-katalysierten Umsetzung von $2 \mathrm{H}$ Azirinen mit enolisierbaren Ketonen [21] darstellt (Schema 6, 20-21).

Schema 6

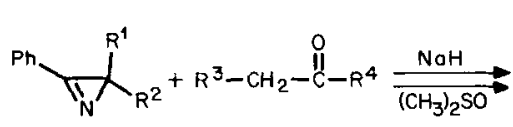

18

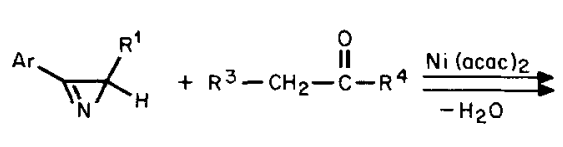

20<smiles>[Z9]C1=NC([R7])([R7])C(c2ccccc2)=C1[Hg]</smiles>

19

21

6) Unter der Einwirkung von Ửbergangsmetallkomplexen kann jede der drei Azirinbindungen gebrochen werden: So erfolgt z.B. die Spaltung der C,N-Doppelbindung ausser bei den in dieser Arbeit beschriebenen Reaktionen auch in Gegenwart von $\mathrm{CO}_{2}(\mathrm{CO})_{8}$ [22], während in 2-Formyl-, 2-Imino- und 2-Vinylazirinen bei der Umsetzung mit $\mathrm{Mo}(\mathrm{CO})_{6}$ die Spaltung der $\mathrm{C}, \mathrm{C}$-Bindung eintritt [19]. Bevorzugt ist aber offenbar die Spaltung der C,N-Einfachbindung, die durch Fe- [1] [23], Rh- [24] und Pd-Komplexe [25] [26] bewirkt wird. 
Schliesslich sind in Schema 7 die drei verschiedenen Azirin-Ringöffnungen einander am Beispiel der Umsetzung mit Acetylendicarbonsäure-dimethylester (2a) gegenübergestellt. Die Reaktion in Gegenwart von $\mathrm{Mo}(\mathrm{CO})_{6}$ führt unter Spaltung der N(1), C(3)-Bindung des Azirins 22 (Weg a)) zu 2 H-Pyrrol-4, 5-dicarbonsäureDerivaten vom Typ 5, Photolyse liefert unter Ringöffnung zwischen C (2) und C (3) (Weg b)) die zu 5 isomeren $2 H$-Pyrrol-3,4-dicarbonsäure-Derivate 6 [5] und die rein thermische Umsetzung des 2-Äthyl-3-dimethylamino-2-phenyl-2 $H$-azirins ergibt unter Spaltung der N(1), C(2)-Bindung das $3 H$-Pyrrol-4, 5-dicarbonsäureDerivat 17 [14].

\section{Schema 7}

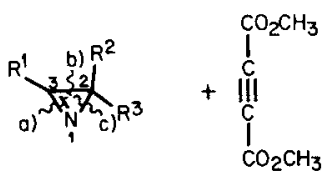

22

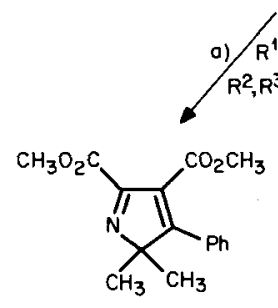

50
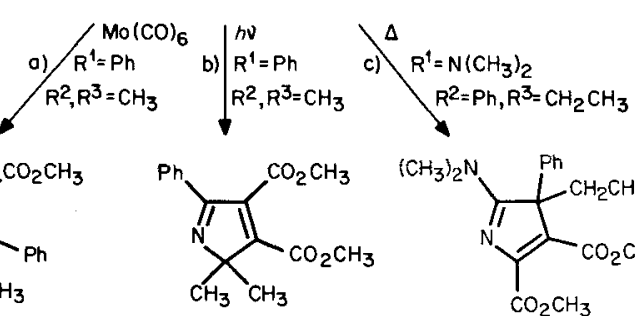

60

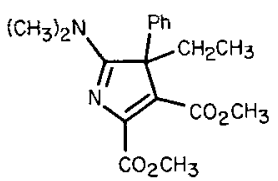

17

Die in der vorliegenden, von Herrn Prof. Dr. Hans Schmid $\dot{\dagger}^{\dagger}$ angeregten Arbeit beschriebenen Versuche sind im Jahre 1977 ausgeführt worden.

Wir danken den analytischen Abteilungen unseres Institutes für die Aufnahme der Spektren und die Ausführung der Analysen. Dem Schweizerischen Nationalfonds zur Förderung der wissenschaftlichen Forschung sowie der F. Hoffmann-LaRoche \& $C o$. $A G$, Basel sei für finanzielle Unterstützung gedankt.

\section{Experimenteller Teil}

Allgemeine Bemerkungen. Abdampfoperationen im Rotationsverdampfer (RV.) bei ca. 12 Torr, Destillationen kleiner Substanzmengen in einem Kugelrohr (Luftbadtemp.). Analytische Dünnschichtchromatogramme (DC.) an Polygram-Kieselgel-Folien, präparative Dünnschichtchromatographie (präp. DC.) an Kieselgel-60- $F_{254}$-Fertigplatten der Firma Merck. Säulenchromatographie an Kieselgel 60 (Merck). Smp. wurden auf einem Mettler-FP-2-Gerät bestimmt. - UV.-Spektren, wenn nicht anders angegeben, in Methanol, Angabe von $\lambda_{\text {max }}$ in $\mathrm{nm}(\log \xi)$. - IR.-Spektren in KBr, Angaben in $\mathrm{cm}^{-1}$.${ }^{1} \mathrm{H}-\mathrm{NMR}$.-Spektren in $\mathrm{CDCl}_{3}$ bei 60 oder $100 \mathrm{MHz},{ }^{13} \mathrm{C}-\mathrm{NMR}$.-Spektren bei 25,2 MHz; chemische Verschiebungen (Bereiche oder Signalzentren) in ppm relativ zu Tetramethylsilan als internem Standard $(=0 \mathrm{ppm})$, Kopplungskonstanten $J$ in $\mathrm{Hz} ; s=$ Singulett, $d=$ Dublett, $t=$ Triplett, $q a=$ Quadruplett, $m=$ Multiplett. - Massenspektren (MS.) an einem CEC-21-110B-Gerät bei $70 \mathrm{eV}$, Angabe der Pike in $m / z$ (rel.-\%).

1. Umsetzung von 2,2-Dimethyl-3-phenyl-2 H-azirin (1) und Acetylendicarbonsäure-dimethylester (2a) in Gegenwart von $\mathrm{Mo}(\mathrm{CO})_{6}$. Eine Suspension von $1,0 \mathrm{~g}(6,9 \mathrm{mmol}) \mathbf{1}, 2,0 \mathrm{~g}(14 \mathrm{mmol}) 2 \mathrm{a}$ und $1,8 \mathrm{~g}$ 
$(6,8 \mathrm{mmol}) \mathrm{Mo}(\mathrm{CO})_{6}$ in $10 \mathrm{ml}$ trockenem Benzol wurde 3 Tage bei $\left.35-45^{\circ 7}\right)$ unter Ar gerührt. Nach dem Abkühlen wurde filtriert und der Rückstand mehrmals mit Benzol gewaschen. Das Filtrat wurde i.RV. eingedampft und an Kieselgel mit Benzol und dann mit Benzol/Äther 1:1 chromatographiert. Die (Benzol/Äther)-Fraktion wurde eingedampft, mit Benzol/Aceton 95:5 erneut chromatographiert und aus Äther umkristallisiert: $560 \mathrm{mg}(28,4 \%$ bzgl. 1) 2,2-Dimethyl-3-phenyl-2 H-pyrrol-4,5-dicarbonsäure-dimethylester (5a), farblose Plättchen vom Smp. 103,5-105º̈ (Äther). - UV.: 293 (3,57), $227(3,58)$; min. 260 (3,47). - IR.: 2990, 2980, 2960, 1750 (Ester-CO), 1715 (Ester-CO), 1625, 1558, 1495, 1445, $1380,1370,1325,1240,1210,1175,1160,1060 .-{ }^{1} \mathrm{H}-\mathrm{NMR}: 7_{7,55-7,05}(\mathrm{~m}, 5$ arom. H); 4,01 und 3,71 $\left(2 s, 2 \mathrm{CO}_{2} \mathrm{CH}_{3}\right) ; 1,50\left(s,\left(\mathrm{CH}_{3}\right)_{2} \mathrm{C}\right) .{ }^{13} \mathrm{C}-\mathrm{NMR} .: 177,0(s, \mathrm{C}(5)) ; 163,6,162,7$ und $160,7\left(3 s, 2 \mathrm{CO}_{2} \mathrm{CH}_{3}\right.$ und $\mathrm{C}(3)) ; 132,7$ ( $s$, arom. C); $128,8,128,3$ und 126,9 ( $3 d$, arom. $\mathrm{C}) ; 126,9(s, \mathrm{C}(4)) ; 82,3(s, \mathrm{C}(2))$; 52,8 und $52,0\left(2 q a, 2 \mathrm{CO}_{2} \mathrm{CH}_{3}\right) ; 22,3\left(q a,\left(\mathrm{CH}_{3}\right)_{2} \mathrm{C}\right),-\mathrm{MS} .: 287\left(60, \mathrm{M}^{+}\right), 272(100), 256(14), 240(13)$, 228 (7), $212(10), 129$ (8), 128 (16), 127 (12), 105 (60). - Osmometrische Mol.-Gew.-Bestimmung $\left(\mathrm{CHCl}_{3}\right)$ : Gefunden 297.

\section{$\mathrm{C}_{16} \mathrm{H}_{17} \mathrm{NO}_{4}(287,30) \quad$ Ber. C 66,86 H 5,96 N 4,88\% $\quad$ Gef. C 66,58 H 5,91 N 5,10\%}

2. Umsetzung von 1 mit Acetylendicarbonsäure-diäthylester (2b) in Gegenwart von $\mathrm{Mo}(\mathrm{CO})_{6}$. Wie in Kap. I beschrieben, wurden $0,7 \mathrm{~g}(4,8 \mathrm{mmol}) \mathbf{1}, 1,6 \mathrm{~g}(9,6 \mathrm{mmol}) 2 \mathrm{~b}$ und $1,3 \mathrm{~g}(4,8 \mathrm{mmol}) \mathrm{Mo}(\mathrm{CO})_{6}$ in $10 \mathrm{ml}$ Benzol bei $35-45^{\circ}$ umgesetzt. Nach üblicher Aufarbeitung wurde der Rückstand an Kieselgel mit Benzol/Aceton 97:3 chromatographiert. Umkristallisation aus Hexan/Äther ergab $307 \mathrm{mg}(20,2 \%)$ 2,2-Dimethyl-3-phenyl-2 H-pyrrol-4,5-dicarbonsäure-diäthylester (5b), farblose Plättchen vom Smp. 92,5-93 (Hexan/Äther). - UV.: 292 (3,67), $228 S$ (3,72); min. 267 (3,58). - IR.: 2990, 2980, 2940, 1745 (Ester-CO), 1715 (Ester-CO), 1630, 1560, 1492, 1447, 1380, 1345, 1315, 1245, 1195, 1180, 1160, 1060. - ' $\mathrm{H}$-NMR.: 7,6-7,05 (m, 5 arom. H); 4,41 und 4,11 (2 qa, $\left.J=7,2 \mathrm{CO}_{2} \mathrm{CH}_{2} \mathrm{CH}_{3}\right) ; 1,46\left(s,\left(\mathrm{CH}_{3}\right)_{2} \mathrm{C}\right)$; 1,41 und $1,07\left(2 t, J=7,2 \mathrm{CO}_{2} \mathrm{CH}_{2} \mathrm{CH}_{3}\right) .-{ }^{13} \mathrm{C}-\mathrm{NMR}$.: 177,3 (s, C(5)); 162,9, 162,6 und 161,2 (3 $s$, $2 \mathrm{CO}_{2} \mathrm{CH}_{2} \mathrm{CH}_{3}$ und $\left.\mathrm{C}(3)\right)$; 133,0 ( $s$, arom. C); $128,6,128,2$ und 126,9 ( $3 d$, arom. $\left.\mathrm{C}\right) ; 127,2(s, \mathrm{C}(4))$; $82,1(s, \mathrm{C}(2)) ; 62,0$ und $60,9\left(2 t, 2 \mathrm{CO}_{2} \mathrm{CH}_{2} \mathrm{CH}_{3}\right) ; 22,1\left(q a,\left(\mathrm{CH}_{3}\right)_{2} \mathrm{C}\right) ; 14,1$ und 13,7 (2qa, $2 \mathrm{CO}_{2} \mathrm{CH}_{2} \mathrm{CH}_{3}$ ), - MS.: 315 (31, M+), 286 (100), 270 (11), 240 (14), 212 (11), 129 (10), 128 (18), $127(12), 105(59)$.

\section{$\mathrm{C}_{18} \mathrm{H}_{21} \mathrm{NO}_{4}(315,36) \quad$ Ber. C 68,55 H 6,71 N 4,44\% Gef. C 68,79 H 6,58 N 4,70\%}

3. Umsetzung von 1 mit Propiolsäuremethylester (2e) in Gegenwart von Mo(CO) ${ }_{6}$. In Analogie zu den in Kap. $I$ und 2 beschriebenen Reaktionen wurde eine Suspension von $1,5 \mathrm{~g}(10,3 \mathrm{mmol}) 1,1,7 \mathrm{~g}$ $(20,2 \mathrm{mmol}) 2 \mathrm{c}$ und $2,7 \mathrm{~g}(10,3 \mathrm{mmol}) \mathrm{Mo}(\mathrm{CO})_{6}$ in $10 \mathrm{ml}$ Benzol $60 \mathrm{Std}$. bei $35-45^{\circ}$ unter Ar gerührt. Nach üblicher Aufarbeitung wurde zuerst mit Aceton/Hexan 1:9 und dann mit Aceton/Hexan 1:4 an Kieselgel chromatographiert. Die mit Aceton/Hexan 1:4 erhaltenen Fraktionen wurden eingedampft und mittels präp. DC. (Kieselgel, $\mathrm{CHCl}_{3}$ ) nachgereinigt. Umkristallisation aus Hexan lieferte $108 \mathrm{mg}$ (5\%) 2,2-Dimethyl-3-phenyl-2H-pyrrol-4-carbonsäure-methylester $(\mathbf{5 c})$, farblose Plättchen vom Smp. 59-61 ${ }^{\circ}$ (Hexan). - UV. (Hexan): 280 Sch. (3,59), 251 (3,60), $230 S$ (3,55). - IR.: 2970, 2920, 1710 (Ester-CO), 1630, 1596, 1485, 1458, 1435, 1375, 1355, 1288, 1245, 1170, 1125, 1005. - ' $\mathrm{H}$-NMR. $\left(\mathrm{CCl}_{4}\right): 8,15(s, \mathrm{H}-\mathrm{C}(5)) ; 7,5-7,0(m, 5$ arom. $\mathrm{H}) ; 3,60\left(s, \mathrm{CO}_{2} \mathrm{CH}_{3}\right) ; 1,32\left(s,\left(\mathrm{CH}_{3}\right)_{2} \mathrm{C}\right) .-\mathrm{MS} .: 229$ $\left(100, M^{+}\right), 214(8), 198(33), 171(12), 170(58), 129(31), 128(15)$.

$$
\mathrm{C}_{14} \mathrm{H}_{15} \mathrm{NO}_{2}(229,27) \quad \text { Ber. C 73,34 H 6,59 N 6,11\% Gef. C 73,14 H 7,09 N 5,88\% }
$$

Aus den mit Aceton/Hexan 1:9 erhaltenen Fraktionen wurden nach Umkristallisation aus Hexan $40 \mathrm{mg}(2,1 \%)$ (E,E)-3, 3'-Oxydiacrylsäure-dimethylester (11) [7] als farblose Nadeln vom Smp. 154-155 erhalten. - UV.: 251,5 (4,1 I). - IR.: 3100, 2970, 1725 (Ester-CO), $1640(C=C), 1445,1345,1235,1210$, $1150,1110,975,865,855 .-1 \mathrm{H}-\mathrm{NMR} .: 7,60(d, J=12, \mathrm{H}-\mathrm{C}(2)) ; 5,67(d, J=12, \mathrm{H}-\mathrm{C}(3)) ; 3,77$ $\left(s, \mathrm{CO}_{2} \mathrm{CH}_{3}\right)$. - MS.: $186\left(16, M^{+}\right), 155(32), 123(20), 116(48), 111(28), 87(76), 85(100)$.

4. Umsetzung von 1 mit Phenylpropiolsäureäthylester (2d) in Gegenwart von $M o(C O)_{6}$. Eine Suspension von $1,5 \mathrm{~g}(10,3 \mathrm{mmol}) 1,3,5 \mathrm{~g}(20,1 \mathrm{mmol}) 2 \mathrm{~d}$ und $1,5 \mathrm{~g}(5,7 \mathrm{mmol}) \mathrm{Mo}(\mathrm{CO})_{6}$ in $10 \mathrm{ml}$ Benzol wurde 3 Tage bei $30-35^{\circ}$ und weitere 3 Tage bei $80-90^{\circ}$ unter Ar gerührt. Nach üblicher Aufarbeitung wurde bei $100-105^{\circ} / 10^{-3}$ Torr destilliert, wobei $3,4 \mathrm{~g} \mathrm{1/2d}$ erhalten wurden. Chromatographie des Rückstandes an Kieselgel mit Benzol und dann mit Äther lieferte als einziges Produkt $102 \mathrm{mg}(6,8 \%)$

7) Bei dieser Temp. war der grösste Teil des $\mathrm{Mo}(\mathrm{CO})_{6}$ in Benzol gelöst. 
2,2,5,5-Tetramethyl-3,6-diphenyl-2,5-dihydropyrazin (8) [6], das durch direkten Vergleich mit dem in Kap. 5 beschriebenen Material identifiziert wurde (DC., Misch-Smp., IR.).

5. Reaktion von 1 in Gegenwart von Mo(CO) 6 . Eine Suspension von $0,5 \mathrm{~g}(3,5 \mathrm{mmol}) 1$ und $1,0 \mathrm{~g}$ $(3,8 \mathrm{mmol}) \mathrm{Mo}(\mathrm{CO})_{6}$ in $7 \mathrm{ml}$ Benzol wurde 5 Tage bei $45-50^{\circ}$ unter Ar gerührt. Nach üblicher Aufarbeitung und Destillation bei $100^{\circ} / 10^{-3}$ Torr wurden $152 \mathrm{mg} 1$ zurückerhalten. Chromatographie des Rückstandes an Kieselgel mit Benzol und anschliessend mit Äther lieferte $127 \mathrm{mg}(25,4 \% ; 36,5 \%$ bzgl. umgesetztem 1$) 8$ [6], farblose Plättchen vom Smp. 126-127 (Hexan). - UV.: 222 (3,61). IR.: $3050,3000,2950,2930,1630,1580,1448,1385,1365,1282,1177,1168,1010,780,720,710,705$. ${ }^{1} \mathrm{H}-\mathrm{NMR}$.: 7,6-7,2 (schmales $m, 10$ arom. H); 1,51 (s, $\left.2\left(\mathrm{CH}_{3}\right)_{2} \mathrm{C}\right)$. - ${ }^{13} \mathrm{C}-\mathrm{NMR}$ : $171,6(\mathrm{C}(3), \mathrm{C}(6))$; $140,3,128,2,128,0$ und 127,4 (arom. C); 56,7 (C(2), C(5)); $29,2\left(2\left(\mathrm{CH}_{3}\right)_{2} \mathrm{C}\right)$. MS. $(20 \mathrm{eV}): 290\left(2, M^{\dagger}\right)$, $260(4,5), 259(6), 187(100), 172(58), 131(35), 116(12), 115(14), 104(15), 103(17), 91$ (18).

\section{$\mathrm{C}_{20} \mathrm{H}_{22} \mathrm{~N}_{2}(290,39) \quad$ Ber. C 82,72 H 7,64 N 9,65\% Gef. C 82,49 H 7,42 N 9,68\%}

6. Umsetzung von 1 mit $2 \mathrm{a}$ in Gegenwart von $\mathrm{WCl}_{6} /\left(\mathrm{C}_{4} \mathrm{H}_{9}\right)_{4} \mathrm{Sn}$. Eine Lösung von $0,5 \mathrm{~g}(3,5 \mathrm{mmol}) \mathbf{1}$, $1,0 \mathrm{~g}(7,0 \mathrm{mmol}) 2 \mathrm{a}, 0,1 \mathrm{~g}(0,3 \mathrm{mmol})$ Tetrabutylzinn und $0,1 \mathrm{~g}(0,25 \mathrm{mmol}) \mathrm{WCl}_{6}$ in $20 \mathrm{ml}$ Trichloräthylen wurde $24 \mathrm{Std}$. bei $45-50^{\circ}$ unter Ar gerührt. Filtration, Waschen des Rückstandes mit Aceton, Eindampfen der Acetonphasen, Chromatographie an Kieselgel mit Methanol/Benzol 3:97 und Nachreinigung mittels präp. DC. $\left(\mathrm{CHCl}_{3}\right)$ ergaben $120 \mathrm{mg}(12 \%) 5 \mathbf{a}$, das durch direkten Vergleich mit dem in Kap. 1 beschriebenen Material identifiziert wurde (DC., Misch-Smp., IR.).

7. Umsetzung von 2,3-Diphenyl-2 $\mathrm{H}$-azirin (7) mit 2a in Gegenwart von $\mathrm{Mo}(\mathrm{CO})_{6}$. Eine Suspension von $1,0 \mathrm{~g}(5,2 \mathrm{mmol}) 7,1,4 \mathrm{~g}(9,9 \mathrm{mmol}) 2 \mathrm{a}$ und $1,3 \mathrm{~g}(5,2 \mathrm{mmol}) \mathrm{Mo}(\mathrm{CO})_{6}$ in $10 \mathrm{ml}$ Benzol wurde $62 \mathrm{Std}$. bei $45-50^{\circ}$ unter Ar gerührt. Nach üblicher Aufarbeitung wurde der Rückstand an Kieselgel nacheinander mit Benzol, Äther und $\mathrm{CHCl}_{3}$ chromatographiert. Die Benzolfraktion enthielt $212 \mathrm{mg} 7$, die Ätherfraktion ergab nach Abdampfen von überschüssigem 2a und Umkristallisation des Rückstandes aus $\ddot{A}$ ther $360 \mathrm{mg}(20,4 \% ; 26,2 \%$ bzgl. umgesetztem 7) 4,5-Diphenylpyrrol-2,3-dicarbonsäuredimethylester (9) [8], farblose Nadeln vom Smp. 186-187 (Äther). - UV.: 297 (4,07), 233 (4,07); min. 261 (3,89). - IR.: 3295 (NH), 3070, 3030, 3005, 2960, 1735 (Ester-CO), 1685 (Pyrrol), 1610, 1520, $1482,1455,1447,1424,1355,1318,1295,1255,1220,1205,1176,1105,1070,995,947,775,705,695$. ${ }^{1} \mathrm{H}$-NMR.: 9,50 (br. $s$, HN; austauschbar mit $\mathrm{D}_{2} \mathrm{O}$ ); 7,4-7,2 (schmales $m, 5$ arom. $\mathrm{H}$ ); 3,85 und 3,75 $\left(2 s, 2 \mathrm{CO}_{2} \mathrm{CH}_{3}\right)$. - MS.: $335\left(69, M^{+}\right), 303$ (100), 272 (16), 271 (10), 270 (19), 245 (22), 244 (18), 218 (11), 217 (41), 216 (27), 215 (15), 214 (24), 189 (22), 178 (19), 107,5 (17), 105 (10), 94,5 (12).

\section{$\mathrm{C}_{20} \mathrm{H}_{17} \mathrm{NO}_{4}(335,34) \quad$ Ber. C 71,63 $\quad \mathrm{H} 5,11 \quad \mathrm{~N} \mathrm{4,18 \%} \quad$ Gef. C 71,49 $\quad$ H 5,27 $\quad$ N 3,94\%}

8. Reaktion von 7 in Gegenwart von $\mathrm{Mo}(\mathrm{CO})_{6}$. Eine Suspension von $0,3 \mathrm{~g}(1,6 \mathrm{mmol}) 7$ und $0,6 \mathrm{~g}$ $(2,3 \mathrm{mmol}) \mathrm{Mo}(\mathrm{CO})_{6}$ in $7 \mathrm{ml} \mathrm{Benzol}$ wurde $65 \mathrm{Std}$. unter $\mathrm{N}_{2}$ auf $45-55^{\circ}$ erwärmt. Nach üblicher Aufarbeitung und präp. DC. an Kieselgel mit Hexan wurden $100 \mathrm{mg} 7$ und $56 \mathrm{mg}$ (18\%; 27\% bzgl. umgesetztem 7) 2,3,5,6-Tetraphenylpyrazin (10) [10] isoliert. Letzteres wurde durch direkten Vergleich (DC., Misch-Smp., IR.) mit authentischem Material identifiziert.

9. ${ }^{13} \mathrm{C}-\mathrm{NMR}$.-Spektren von $2 \mathrm{H}$-Pyrrolen. - 9.1. 2,2-Dimethyl-5-phenyl-2H-pyrrol-3, 4-dicarbonsäuredimethylester (6a) [5]. - ${ }^{13} \mathrm{C}-\mathrm{NMR} .: 165,4,165,1,161,5$ und $159,9\left(2 \mathrm{CO}_{2} \mathrm{CH}_{3}, \mathrm{C}(3), \mathrm{C}(5)\right) ; 137,3(\mathrm{C}(4))$; $133,0,130,4,128,5$ und 127,5 (arom. C); 79,3 $(\mathrm{C}(2)) ; 52,6$ und 52,2 $\left(2 \mathrm{CO}_{2} \mathrm{CH}_{3}\right) ; 22,9\left(\left(\mathrm{CH}_{3}\right)_{2} \mathrm{C}\right)$.

9.2. 2,2-Dimethyl-4,5-diphenyl-2H-pyrrol-3-carbonsäureäthylester (14) [15]. - ${ }^{13} \mathrm{C}$-NMR : 169,2, 163,2 und $157,0\left(\mathrm{CO}_{2} \mathrm{CH}_{2} \mathrm{CH}_{3}, \mathrm{C}(3), \mathrm{C}(5)\right) ; 144,5(\mathrm{C}(4)) ; 133,7,133,6,129,4,128,8,128,6,127,8$ und 127,6 (arom. C); 78,8 (C(2)); 60,3 ( $\left.\mathrm{CO}_{2} \mathrm{CH}_{2} \mathrm{CH}_{3}\right) ; 23,6\left(\left(\mathrm{CH}_{3}\right)_{2} \mathrm{C}\right) ; 13,7\left(\mathrm{CO}_{2} \mathrm{CH}_{2} \mathrm{CH}_{3}\right)$.

9.3. $\quad 5-(\mathrm{N}$-Methylanilino)-2,2-dimethyl-2H-pyrrol-3, 4-dicarbonsäure-dimethylester (15) [28]. ${ }^{13} \mathrm{C}-\mathrm{NMR}$ : $163,9,161,8,159,6$ und 158,9 $\left(2 \mathrm{CO}_{2} \mathrm{CH}_{3}, \mathrm{C}(3), \mathrm{C}(5)\right) ; 144,9$ (arom. C); 133,7 (C(4)); $129,0$ und 126,0 (arom. $\mathrm{C}) ; 73,9(\mathrm{C}(2)) ; 52,0$ und 51,6 $\left(2 \mathrm{CO}_{2} \mathrm{CH}_{3}\right) ; 41,3\left(\mathrm{CH}_{3} \mathrm{~N}\right) ; 24,6\left(\left(\mathrm{CH}_{3}\right)_{2} \mathrm{C}\right)$.

\section{LITERATURVERZEICHNIS}

[1] Y. Nakamura, K. Bachmann, H. Heimgartner, H. Schmid \& J.J. Daly, Helv. Chim. Acta 61, 589 (1978).

[2] A. Inada, Y. Nakamura, H. Heimgartner \& J.J. Daly, unveröffentlichte Ergebnisse.

[3] H. Alper \& J. E. Prickett, Inorg. Chem. 16, 67 (1977); H. Alper, Israel J. Chem. 21, 203 (1981).

[4] A. Inada, H. Heimgartner \& H. Schmid, Tetrahedron Lett. 1979, 2983. 
[5] N. Gakis, M. Märky, H.-J. Hansen, H. Heimgartner, H. Schmid \& W.E. Oberhänsli. Helv. Chim. Acta $59,2149(1976)$.

[6] D. G. Farnum \& G.R. Carlson, Synthesis 1972, 191.

[7] E. Wenkert, K.A.H. Adams \& C.L. Leicht, Can. J. Chem. 41, 1844 (1963).

[8] J. B. Hendrickson, R. Rees \& J. F. Templeton, J. Am. Chem. Soc. 86, 107 (1964).

[9] H. Giezendanner. M. Märky, B. Jackson, H.-J. Hansen \& H. Schmid, Helv. Chim. Acta 55, 745 (1972); A. Padwa, M. Dharan, J. Smolanoff \& S. I. Wetmore, J. Am. Chem. Soc. 95, 1945 (1973).

[10] N. Gakis, M. Märky, H.-J. Hansen \& H. Schmid, Helv. Chim. Acta 55, 748 (1972).

[11] H. Alper \& S. Wollowitz, J. Am. Chem. Soc, 97, 3541 (1975).

[12] U. Widmer, N. Gakis, B. Amet, H. Heimgartner \& H. Schmid, Chimia 30, 453 (1976); vgl. auch W. Stegmann, P. Gilgen, H. Heimgartner \& H. Schmid, Helv. Chim. Acta 59, 1018 (1976).

[13] V. Dave, J.B. Stothers \& E.W. Warnhoff, Tetrahedron Lett. 1973, 4229.

[14] M. Henriet, Dissertation Université Catholique de Louvain 1979.

[15] U. Gerber, H. Heimgartner, H. Schmid \& W. Heinzelmann. Helv. Chim. Acta 60, 687 (1977).

[16] N. Calderon, J.P. Lawrence \& E.A. Ofstead, Adv. Organomet. Chem. 17, 449 (1979); T.J. Katz, ibid. 16, 283 (1977); T.J. Katz \& S.J. Lee, J. Am. Chem. Soc. 102, 422 (1980) und dort zit. Lit.

[17] A. Mortreux \& M. Blanchard, J. Chem. Soc., Chem. Commun. 1974, 786; G. S. Lewandos \& R. Pettit, J. Am. Chem. Soc. 93, 7087 (1971).

[18] R. H. Grubbs \& C. R. Hoppin, J. Chem. Soc., Chem. Commun. 1977, 634; K. Ichikawa \& K. Fukuzumi, J. Org. Chem. 41, 2633 (1976).

[19] H. Alper, J. E. Prickett \& S. Wollowitz, J. Am. Chem. Soc. 99, 4330 (1977).

[20] R. Noyori, in 'Transition Metal Organometallics in Organic Synthesis', Herausgeber H. Alper, Vol. I, Academic Press, New York 1976, S. 83; K.M. Nicholas, M. O. Nestle \& D. Seyferth, in 'Transition Metal Organometallics in Organic Synthesis', Herausgeber H. Alper, Vol. II, Academic Press, New York 1978, S. 1; T. Matsuda, N. Sasaki \& T. Higashima, Macromolecules 8, 717 (1975); R.J. Haines \& G. J. Leigh, Chem. Soc. Rev. 4, 155 (1975).

[21] P.F. dos Santos Filho \& U. Schuchardt. Angew. Chem. 89, 672 (1977).

[22] H. Alper \& J.E. Prickett, Tetrahedron Lett. 1976, 2589.

[23] H. Alper \& J.E. Prickett, J. Chem. Soc., Chem. Commun. 1976, 191.

[24] T. Sakakibara \& H. Alper, J. Chem. Soc., Chem, Commun. 1979, 458.

[25] K. Isomura, K. Uto \& H. Taniguchi, J. Chem. Soc., Chem. Commun. 1977, 664.

[26] H. Alper, Ch. P. Perera \& F. R. Ahmed, J. Am. Chem. Soc. 103, 1289 (1981).

[27] J.M. Patterson, Synthesis 1976, 281.

[28] K. Dietliker, Dissertation Universität Zürich 1980; vgl. auch K. Dietliker, W. Stegmann \& H. Heimgartner, Heterocycles 14,929 (1980).

[29] N. Gakis, H. Heimgartner \& H. Schmid, Helv. Chim. Acta 57, 1403 (1974).

[30] A. Laurent, P. Mison, A. Nafti \& N. Pellissier, Tetrahedron Lett. 1978, 4511; A. Padwa \& Y. Kulkarni, ibid. 1979, 107. 